\title{
Childhood Rhabdomyosarcoma with Mixed Embryonal and Alveolar Features
}

National Cancer Institute

\section{Source}

National Cancer Institute. Childhood Rhabdomyosarcoma with Mixed Embryonal and

Alveolar Features. NCI Thesaurus. Code C7960.

A mixed rhabdomyosarcoma that occurs in children. The neoplasm is composed of embryonic and alveolar components. It is characterized by the presence of spindle cells with myoblastic differentiation and a myxoid and a fibrous stroma. 